\title{
Inclusions Size-based Fatigue Life Prediction Model of NiTi Alloy for Biomedical Applications
}

\author{
Marco Fabrizio Urbano ${ }^{1}$ - Andrea Cadelli ${ }^{1} \cdot$ Frank Sczerzenie $^{2}$. \\ Pietro Luccarelli ${ }^{3} \cdot$ Stefano Beretta $^{3} \cdot$ Alberto Coda $^{1}$
}

Published online: 4 June 2015

(C) ASM International 2015

\begin{abstract}
Current standards consider the size and distribution of inclusions in semi-finished material, but do not place requirements on final biomedical devices made of NiTi shape memory alloys. In this paper, we analyze this by comparing the fatigue performances of NiTi superelastic wires obtained by different processes through a simple bilinear model of fatigue response in terms of strain life. The fracture surfaces of failed wires are analyzed through SEM microscopy and data regarding the presence of particles, and their morphology is recorded and analyzed using Type-I extreme value distribution. The results show a strong correlation between the fatigue limit of wires (in terms of strain) and the predicted extreme values of inclusions at fracture origin. Then, following the concept of treating the inclusions as 'small cracks,' a simple relationship between fatigue limit strain range and inclusion size is proposed based on $\Delta \mathrm{K}_{\mathrm{th}}$ data from the literature. The model is compared with the fatigue data obtained from the tested wires.
\end{abstract}

Keywords NiTi superelastic wires · Fatigue - Inclusions · Extreme value $\cdot \Delta \mathrm{K}_{\mathrm{th}} \cdot$ Kitagawa diagram

Alberto Coda

alberto_coda@saes-group.com

1 SAES Getters S.p.A., Lainate, Italy

2 SAES Smart Materials, New Hartford, NY, USA

3 Mechanical Engineering, Politecnico of Milano, Milan, MI, Italy

\section{Introduction}

Since 1979 with the studies of Melton and Mercier [1], significant efforts have been devoted to the study of the fatigue behavior on NiTi shape memory alloys (SMA) for biomedical applications (typically known as Nitinol). The reader can refer to the review by Adler et al. in 2007 [2] and the more recent paper by Pelton et al. [3] to have an overview on fatigue studies of superelastic Nitinol and the difficulties related to this peculiar material. The type of annealing, the nickel/titanium balance, and the relative amount of martensite and austenite are just some of many parameters potentially affecting fatigue behavior of Nitinol that have been considered to date.

In Reinoehl et al. [4], the rotary beam fatigue testing (RBT) of 267- $\mu \mathrm{m}$ diameter wires obtained from different suppliers is characterized by different maximum inclusion dimensions, but no conclusive statements are provided. In Sawaguchi [5] inclusions, scratches and surface defects are documented as nucleation sites of fatigue cracks and in Wong [6] inclusions are recognized as key contributors to failure for as-drawn or electro-polished Nitinol wires. More recently, significant progresses were done to systematically study and clarify the effects of inclusions on the fatigue performance of NiTi SMA [7-10]. Nevertheless, the limited knowledge about the effect of inclusions is still reflected in the regulations pertinent to the characterization of inclusions in Nitinol. The inclusion rating is covered only in standard specification ASTM F2063-12 [11] that requires the evaluation of microcleanliness in wrought Nickel-Titanium SMA for Medical Devices and Surgical Implants. In this procedure, microcleanliness is evaluated in mill products with section sizes between 5.5 and $94.0 \mathrm{~mm}$ in diameter. These dimensions exclude most of finished components for biomedical applications. Thus, the 
inclusion assessment is performed at an intermediate manufacturing process step.

Quite recently, a standard practice for the statistical analysis of nonmetallic inclusions in steels was introduced [12]. The aim of the practice is to estimate the expected largest inclusion in a determined volume of material. The need for a thorough method for the analysis of inclusions followed the studies reported in [13], focused in particular on ball bearing steels that demonstrate the negative effect of inclusions on the fatigue behavior of hard steels and the need to adopt the so called extreme value inclusion rating.

In this work, we present the results of a research program aimed at verifying whether these findings are applicable to Nitinol.

Several lots of superelastic wires manufactured by different melting techniques and hot processing steps were selected. Although presenting almost identical surface finishes and thermo-mechanical properties, the process differences are hypothesized to influence the inclusion nature, size, and distribution (verified a posteriori). Wires are Rotary beam tested (RBT) at several strain levels and fracture sections observed by field emission scanning electron microscopy (FESEM), measuring the dimensions of the inclusions present at the fracture nucleation sites. The negative effect of inclusions on fatigue, independent of process, is demonstrated. The entire set of fatigue data collected for each process is analyzed through an interpretative model, namely a bilinear uniform scatter band model. This analysis allows appreciating quantitatively how different processes can influence the fatigue behaviors, in particular, in the low-strain/long-life region. Similarly, the statistical distribution of the recorded inclusion sizes for each process is considered. The populations are found to follow the Gumbel distribution and to be different, thus confirming the initial assumption. Moreover, the size of extreme inclusions in each material is correlated to its fatigue limits calculated from the experimental tests.

Finally, considering the inclusions as 'small cracks,' a simple relationship between fatigue limit strain range and inclusion size based on $\Delta K_{\text {th }}$ data from the literature can be proposed. The relationship between $\mathrm{d} a / \mathrm{d} N-\Delta K$ is then integrated for estimating the fatigue life. The model is compared with wire fatigue data demonstrating a very good agreement.

\section{Experimental}

\section{Materials}

The 300- $\mu \mathrm{m}$ superelastic wires used in this study come from eight Ni-44.0Ti wt\% ingots and several lots manufactured by means of five different processes, referenced as $\mathrm{P} 1, \mathrm{P} 2, \mathrm{P} 3, \mathrm{P} 4$, and $\mathrm{P} 5$. The processes differ in the raw materials and melting practice, through varied combinations of vacuum induction melting (VIM) and vacuum arc re-melting (VAR), and in the hot processing steps. Cold drawing, interpass annealing, surface polishing, and final straight annealing have been carried out according to a standard recipe. The final surface finish is an amber oxide for all the wires. This surface finish was selected because it is a standard for commercial wires.

Every material was characterized at ingot level in terms of gas impurities (C, O) by a LECO analyzer (ASTM F2063 [11]). Differential scanning calorimetric (DSC) analysis was performed on final annealed wires according to ASTM F2004 [14], as well as bend and free recovery (BFR) and tensile test were performed according to ASTM F2082 [15] and F2516 [16], respectively. Results summarized in Table 1 are the average of three repetitions from each sample. For this experimental campaign, wires having very similar thermo-mechanical properties were selected, in order to minimize the impact of further differences on fatigue.

Metallographic longitudinal centerline samples of wires from the five different processes were prepared by standard practice and analyzed by SEM. In Fig. 1a-e, back-scattered secondary electron (BSE) SEM images of the five materials are reported. The pictures show that the main differences between the materials are due to a different nature and dimensional distribution of the inclusions. The preliminary inclusions analysis was conducted according to the internal procedure described by Sczerzenie et al. [17]. In particular, the samples were taken from head, center, and tail of full size production spools. The analysis was carried out by scanning the length of each sample in three regions of the cross-section: the centerline, the mid-radius, and the near edge lines. Nine fields of view and over one hundred particles per sample were analyzed. Table 2 summarizes the results in terms of maximum area fraction and inclusion size both for carbides and oxides as recommended by ASTM F2063.

Material P1 shows a mix of carbides and oxides, P2 and P3 only carbides, while P4 and P5 exhibit mainly oxides. Although all wires have the same surface finish and undergo the same cold drawing and annealing process, it is not possible to completely exclude other microstructural differences, such as grain size.

\section{Fatigue Testing}

Almost 1500 samples were fatigue tested in zero-mean strain conditions $(R=-1)$ by means of RBT at different alternating strain amplitudes. A rotating beam Blockwise Fatigue Tester FTX was used. All tests were performed at $1000 \mathrm{rpm}$ in a temperature-controlled water bath at $37^{\circ} \mathrm{C}$. 
Table 1 Chemical and mechanical properties of the tested wires

\begin{tabular}{|c|c|c|c|c|c|c|c|c|c|c|}
\hline \multicolumn{2}{|c|}{ Material } & \multicolumn{2}{|c|}{ Impurities (ppm) } & \multicolumn{2}{|c|}{$\frac{\mathrm{TTR}\left({ }^{\circ} \mathrm{C}\right)}{\text { Wire }}$} & \multicolumn{5}{|c|}{ Mechanical properties (at $37^{\circ} \mathrm{C}$ ) } \\
\hline Heat & Lots & Carbon & Oxygen & $A_{\mathrm{f}}$ & $A_{\mathrm{f}}^{\mathrm{BFR}}$ & UTS (MPa) & $\varepsilon_{\max }$ & $\varepsilon_{\mathrm{res}}$ & UPS (MPa) & LPS (MPa) \\
\hline P1 & 2 & 265 & 211 & 14 & 3 & 1335 & 12.2 & 0.22 & 524 & 323 \\
\hline $\mathrm{P} 2$ & 4 & 252 & 247 & 13 & 2 & 1332 & 12.3 & 0.17 & 543 & 339 \\
\hline P3 & 1 & 250 & 250 & 16 & 4 & 1313 & 11.3 & 0.10 & 553 & 355 \\
\hline P4 & 1 & $<20$ & 210 & 13 & 2 & 1333 & 12.6 & 0.22 & 547 & 354 \\
\hline P5 & 1 & $<20$ & $<50$ & 16 & 0 & 1292 & 11.3 & 0.20 & 536 & 340 \\
\hline
\end{tabular}
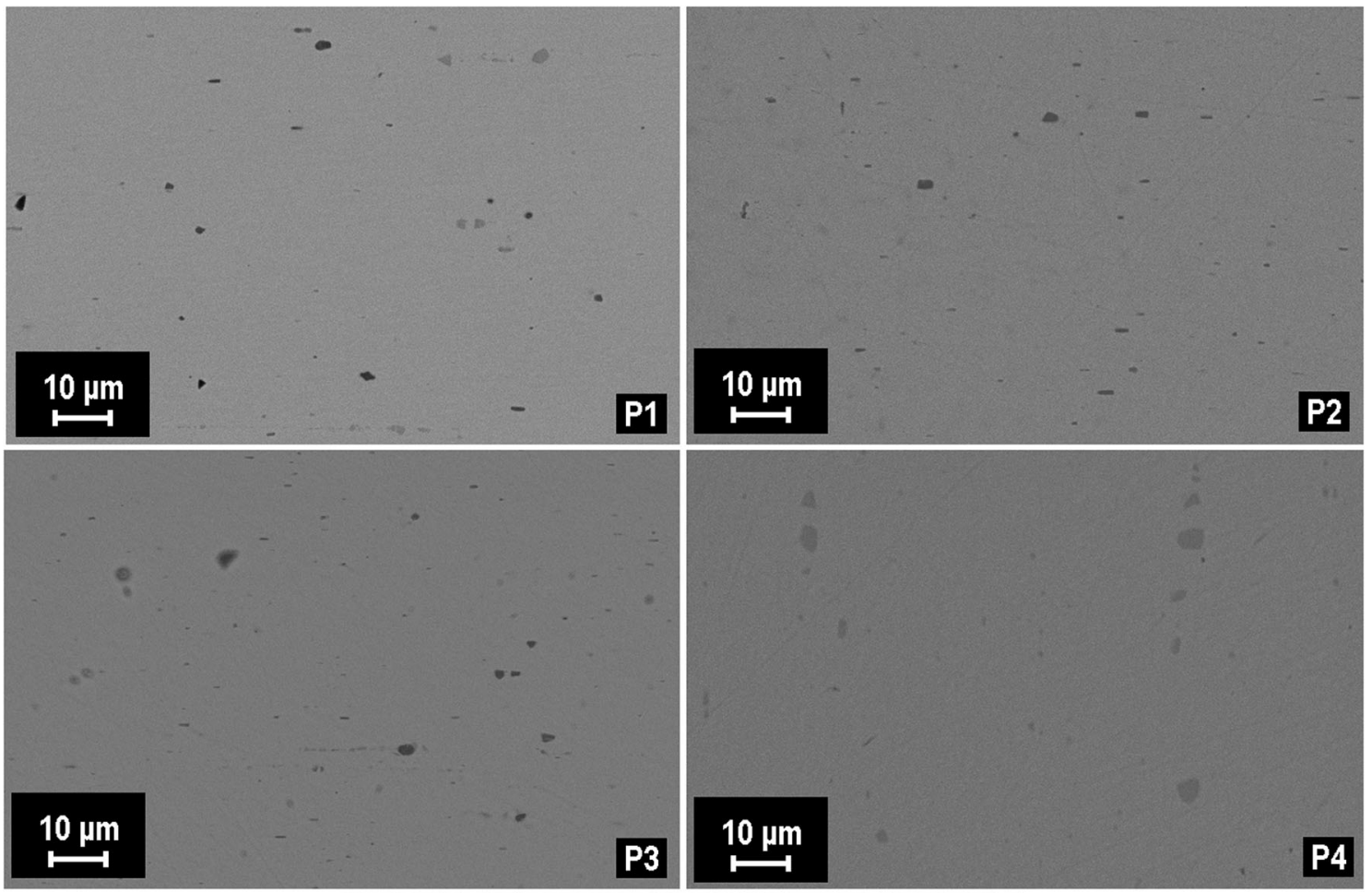

\section{$10 \mu \mathrm{m}$}

1

Fig. 1 BSE SEM images of longitudinal metallographic sections of wires from processes P1-P5. Black particles are titanium carbide (TiC) inclusions and gray particles are intermetallic oxides (TixNiyOz)

Eight to fifteen tests were repeated for each deformation and a run-out criterion was fixed at 1 million cycles. Some tests, however, were extended further with run-outs defined as 24 million cycles.
More than 800 failed wires were analyzed by SEM. A typical fatigue crack originating from an inclusion is shown in Fig. 2. The observation of the fracture surfaces allows one to determine whether an inclusion nucleated the crack 
Table 2 Inclusion analysis performed on metallographic longitudinal cross-section of the five examined NiTi wires according to ASTM F2063

\begin{tabular}{llrlrr}
\hline Material & \multicolumn{2}{l}{$\begin{array}{l}\text { Max area } \\
\text { fraction }(\%)\end{array}$} & & & \multicolumn{2}{l}{$\begin{array}{l}\text { Max inclusion } \\
\text { size }(\mu \mathrm{m})\end{array}$} \\
\cline { 2 - 3 } \cline { 6 - 6 } & Carbides & Oxides & & Carbides & Oxides \\
\hline P1 & 0.73 & 0.15 & & 6.33 & 7.18 \\
P2 & 0.47 & $<0.01$ & & 7.07 & $<0.10$ \\
P3 & 0.73 & 0.04 & & 14.06 & 3.83 \\
P4 & 0.02 & 0.91 & & 2.65 & 34.31 \\
P5 & 0.02 & 0.28 & & 3.14 & 10.61 \\
\hline
\end{tabular}

and, when present, the dimensions of the inclusion at the nucleation site. From SEM analysis, all the fractures nucleate from an inclusion. In particular, in materials where carbides are the primary inclusions, more than $80 \%$ of fractures showed a carbide at the crack tip. In materials where oxides are the dominant inclusions, $100 \%$ of fractures nucleate from these inclusions that, in general, are larger than carbides. The nature of inclusions (carbide or oxide) was also identified and recorded by morphology and chemical composition by using EDX microprobe. The distance of the particle from the surface and its shape were also measured in order to properly compensate the particle area as recommended by Murakami [13]. Measurements of inclusions size were expressed in terms of Murakami's $\sqrt{ }$ area parameter.

Although RBT is an excellent tool for rapidly assessing fatigue performance, the trends presented and discussed in this work may not necessarily be correlated to those that could be observed in finished devices that utilize Nitinol. The effect of inclusions is clearly demonstrated, but readers should assess fatigue testing on their own devices using the relevant deformation mode and frequency.

\section{Data Analysis}

The typical results of rotary beam fatigue tests can be plotted as $\Delta \varepsilon-N$ diagrams, similar to the $\mathrm{S}-\mathrm{N}$ diagrams usually adopted in load-controlled fatigue. Considering that the experimental plan was aimed at determining the fatigue limit in the long-life region, a statistical analysis to describe both fatigue regions (namely 'finite life' and 'fatigue limit') was adopted.

In particular, a bilinear uniform scatter band (firstly proposed by Haibach [18]) was chosen, as is common in establishing design curves for components subjected to fatigue (see, for example, Eurocode 3 curves for welded joints). The simple assumption behind the method is that, in a $\log \mathrm{S}-\log \mathrm{N}$ diagram, a fatigue response can be described by means of a statistical model with constant dispersion along the $\log S$ direction. This method can be applied only to those sets of data which the $\log \mathrm{S}-\log \mathrm{N}$ diagram shows a fitting attitude for a two slopes curve, namely the dataset should contain data also for the fatigue limit region.

The same concept was adopted for the analysis of the present $\Delta \varepsilon-N$ data. Consequently, the following hypotheses (that are consistent with the ASTM E739 recommendations [19]) were included in the statistical model (see Fig. 3):

- Fatigue life, that is the dependent variable in the fatigue experiments, is assumed to be log-normally distributed;

- The dependence of fatigue life on $\Delta \varepsilon$ is modeled as

$$
\begin{array}{ll}
\mu_{\log N}=\log N_{\mathrm{D}}+k_{1} \times\left(\log \Delta \varepsilon-\log \Delta \varepsilon_{D}\right) & \text { for } \varepsilon>\varepsilon_{\mathrm{D}} \\
\mu_{\log N}=\log N_{\mathrm{D}}+k_{2} \times\left(\log \Delta \varepsilon-\log \Delta \varepsilon_{D}\right) & \text { for } \varepsilon<\varepsilon_{\mathrm{D}}
\end{array}
$$

where $\left\{N_{\mathrm{D}}, \Delta \varepsilon_{\mathrm{D}}\right\}$ identifies the 'knee' in the fatigue response;
Fig. 2 A typical fracture surface with details of initial propagation region where it can be seen TixNiyOz particle at fracture origin
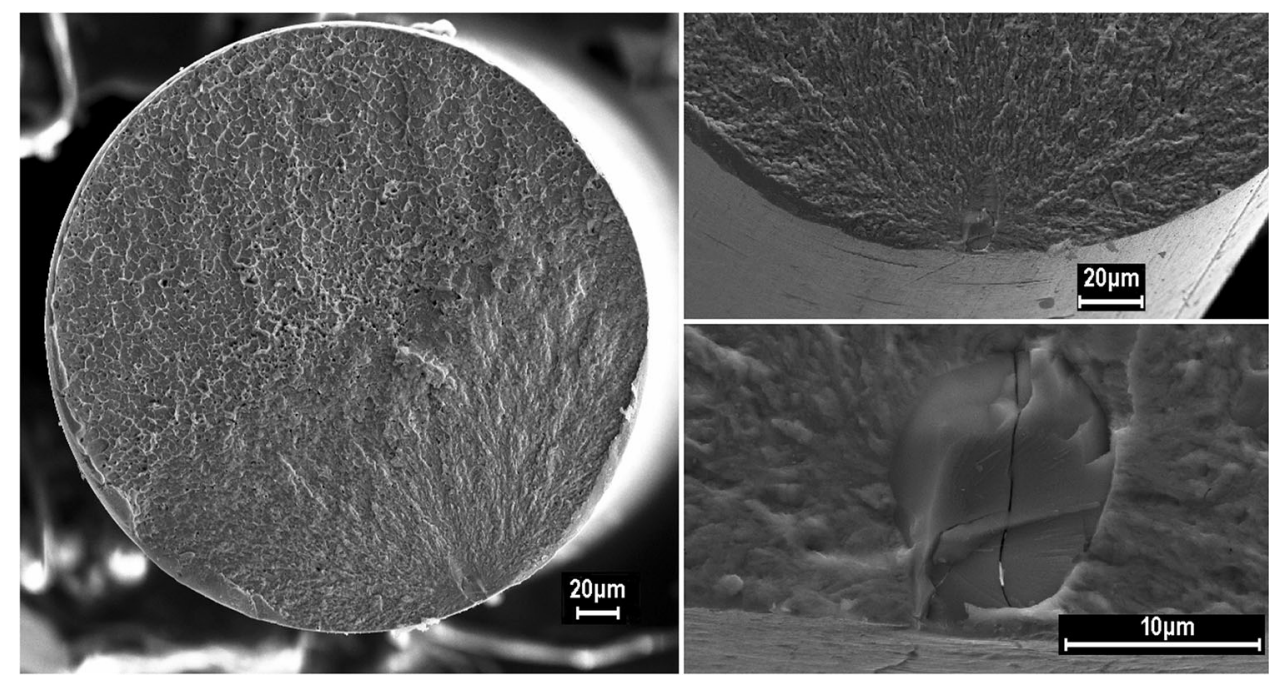


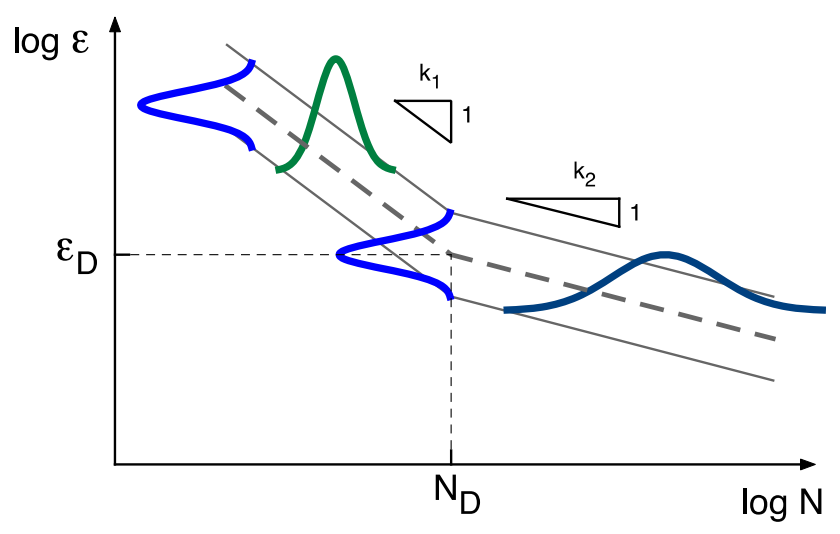

Fig. 3 Concept of the "uniform scatter band" applied to wire rotating bending fatigue data

- In order to model the uniform scatter in the $\log \Delta \varepsilon$ direction, the standard deviation of $\log N$ is described as follows:

$$
\begin{gathered}
\sigma_{\log N}=\sigma_{\log \varepsilon} \times k_{1} \quad \text { for } \varepsilon>\varepsilon_{\mathrm{D}} \\
\sigma_{\log N}=\sigma_{\log \varepsilon} \times k_{2} \quad \text { for } \varepsilon<\varepsilon_{\mathrm{D}}
\end{gathered}
$$

The analysis of the fatigue data for the different materials was conducted with the maximum-likelihood (ML) method [20], maximizing the log-likelihood of the data for a given sample:

$l=\sum_{i=1}^{n} \log \left(f\left(y_{i}\right)\right)+\sum_{j=1}^{u} \log \left(1-F\left(y_{j}^{\prime}\right)\right)$,

where

- $f(y)$ and $F(y)$, respectively, indicate the probability density and the distribution function of the fatigue life $\mathrm{y}$, which are assumed to be a log-normal distribution whose parameters are described by Eqs. $(1,2)$;

- $y_{i}(i=1, \ldots, n)$ and $y_{j}(j=1, \ldots, u)$ indicate a complete test (failure) and a suspended test (run-out), respectively.

Goodness of fit tests proved the adoption of the bilinear model and LR tests for significance of parameters [20] supported the analyses.

The results of such analysis for the five materials are shown in Fig. 4, where it can be seen that the analysis with the bilinear uniform scatter band is able to provide a complete picture of the fatigue behavior of the wires. The fatigue properties that have been considered to be relevant are the strain corresponding to $50 \%$ failure probability at $10^{6}$ cycles, $\varepsilon_{\mathrm{av}}$, and its lower bound (calculated as the $5 \%$ percentile), $\varepsilon_{\text {inf }}$.

A comparison of the model parameters for the five processes is shown in Table 3. It can be seen that the wires with the lowest fatigue limit are the P4's, while the best fatigue limit corresponds to P3 and P5 wires.

\section{Experimental Evidence of Inclusion Effect}

Several inclusion observations at the origin of fracture surface are available for all the samples. To give a qualitative example, the dimension of the inclusions (expressed in terms of the parameter $\sqrt{ }$ area) is reported on the fatigue response for material P1 in Fig. 5. It can be seen that at strain levels near the fatigue limit (e.g., strain levels \pm 0.008 and \pm 0.009 ), the fatigue life is inversely correlated to defect dimension. Alternatively, most of the data points lying on the $5 \%$ line present the largest inclusions. This fact clearly illustrates the detrimental effect of inclusion size on the fatigue limit of NiTi wires, the main topic of the present research.

In the next section, this effect will also be quantified.

\section{Estimation of Extreme Inclusions}

The effect of a prospective population of inclusions/defects in a material subjected to a given stress level is shown in Fig. 6. In particular, the material will fail only if the maximum inclusion is above the $\Delta \sigma_{\text {lim }}-\sqrt{ }$ area relationship. Because of this, fatigue limits are not correlated to the average inclusion content but to the size of the extreme, or maximum, inclusions. A number of successful applications support these concepts (see references [21-23]) that have then been condensed into simple recommendations for the extreme value inclusion rating [ASTM E2283-08] [12].

The estimation of maximum defects is conducted with the statistics of extremes: in the most simple version, it is based on the block maxima sampling, where only the maximum defects detected on a given area (or volume) are recorded and then analyzed with type-I Extreme Value (also called Largest Extreme Value, or Gumbel) Distribution [24]. This sampling strategy can be applied very simply to the inclusion detected at the origin of fatigue failures, since the wires in the central part of the specimen (where they are bent with a constant radius of curvature) are subjected to a constant stress/strain and we expect that failures have occurred at the maximum inclusion present in the most stressed volume.

According to extreme value statistics, the cumulative probability of the particles diameter for each wire batch should follow the Gumbel distribution function [25]:

$F(x)=\exp \left[-\exp \left(-\frac{x-\lambda}{\delta}\right)\right]$

where $\lambda$ and $\delta$ are the location (63.2 percentile) and the scale parameters, respectively. 

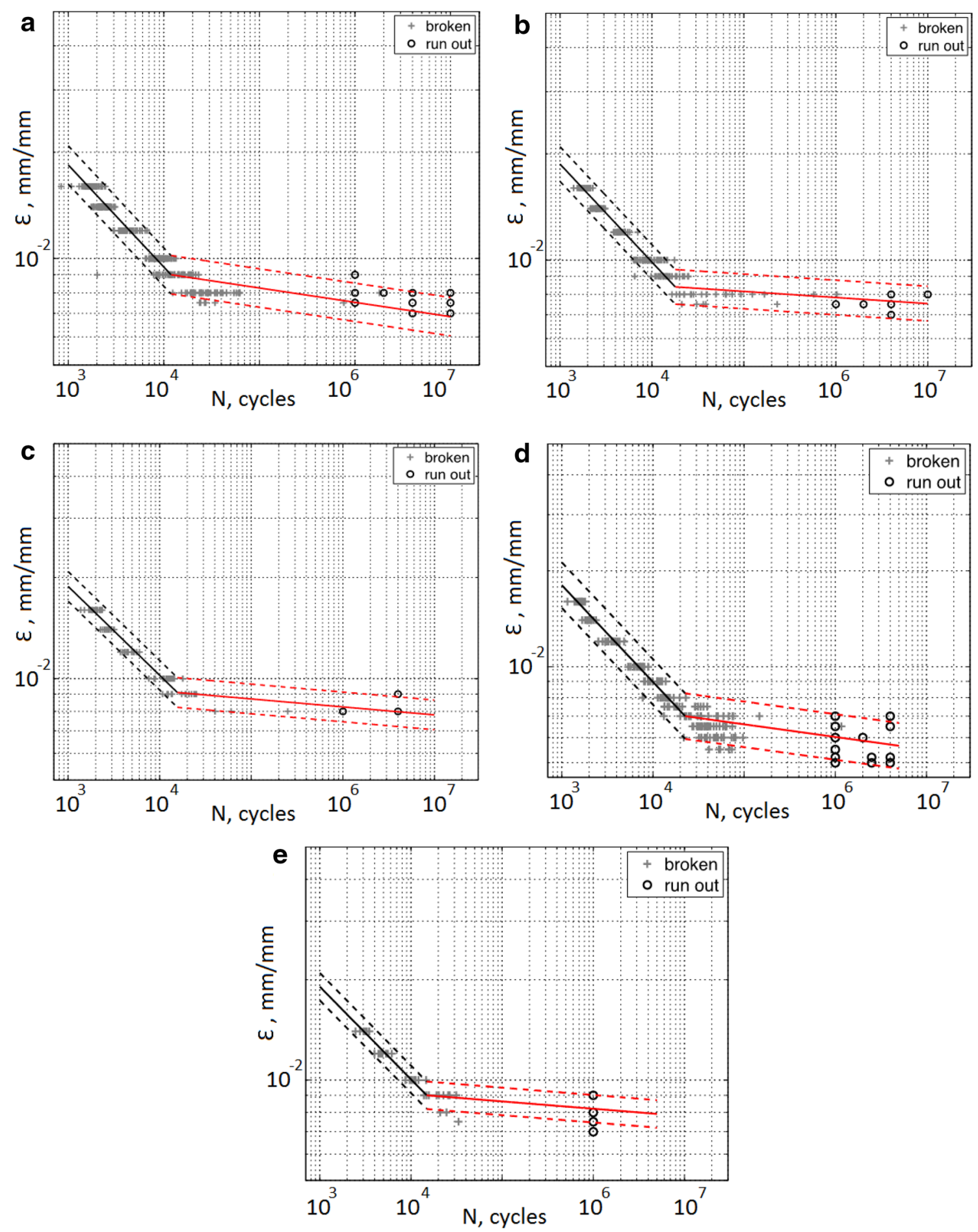

Fig. 4 Interpolation of RBT fatigue data on Nitinol wires: a P1 data; b P2 data; c P3 data; d P4 data; e P5 data

The distribution parameters $\lambda$ and $\delta$ have been estimated as [25]:

$\hat{\delta}=\frac{\sqrt{6}}{\pi} s \quad \hat{\lambda}=\bar{x}+0.5772 \delta$, where $\bar{x}$ is the sample mean of defects dimension and $s$ is the standard deviation of the sampled data.

Using Eq. (4), data can be plotted on a Gumbel probability plot, where the defect inclusions are plotted against 
Table 3 Best-fit parameters of the bilinear uniform scatter band models and fatigue limit expressed in term of strain amplitude at $10^{6}$ cycles

\begin{tabular}{lllllll}
\hline Material & $k_{1}$ & $k_{2}$ & $\Delta \varepsilon_{\mathrm{D}}(\%)$ & $N_{\mathrm{D}}$ & $\Delta \varepsilon_{\mathrm{av}}\left(\%\right.$ at $10^{6}$ cycles $)$ & $\Delta \varepsilon_{\text {inf }}\left(\%\right.$ at $10^{6}$ cycles $)$ \\
\hline P1 & -3.46 & -24.64 & 0.90 & 11,900 & 0.75 & 0.66 \\
P2 & -3.60 & -57.89 & 0.84 & 17,900 & 0.78 & 0.70 \\
P3 & -3.78 & -42.18 & 0.91 & 15,600 & 0.82 & 0.76 \\
P4 & -3.46 & -36.08 & 0.70 & 26,000 & 0.63 & 0.55 \\
P5 & -3.60 & -45.32 & 0.90 & 14,900 & 0.82 & 0.75 \\
\hline
\end{tabular}

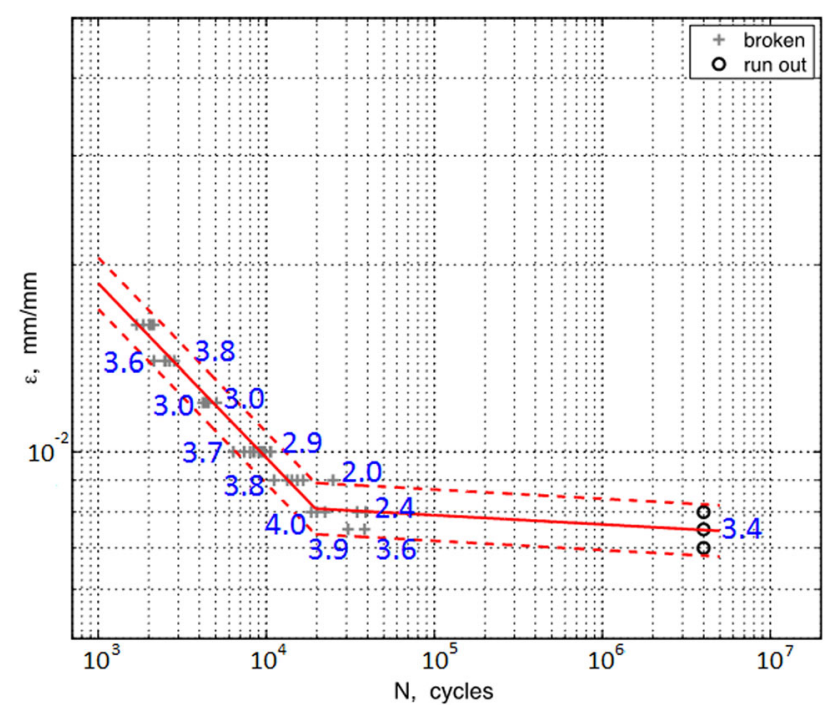

Fig. 5 Empirical correlation between test results and inclusion dimension of the first lot of the P1 wires: inclusion size in micron (expressed in terms of $\sqrt{ }$ area) is reported on the plot aside the relative $\varepsilon-N$ value for most fatigue data

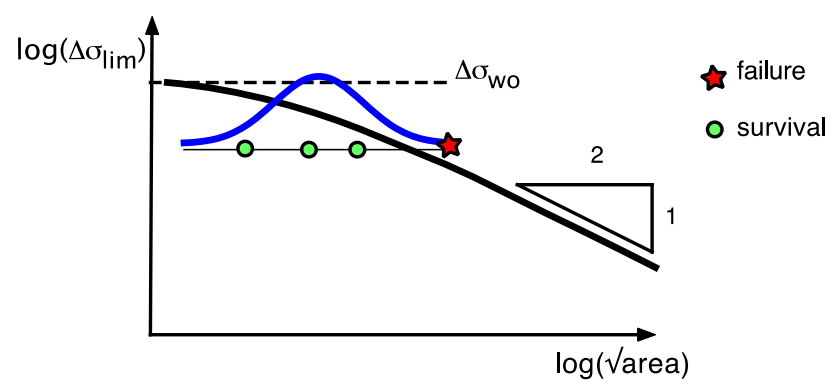

Fig. 6 Schematic of the Kitagawa diagram: for tests at a given stress (or strain) level only the maximum inclusion which is present in the material will lead to failure

the reduced variate $-\ln \left(-\ln \left(F_{i}\right)\right)$, where $F_{i}$ are the empirical cumulative probabilities for the different data points [ASTM E2283-08]. Such a plot is shown in Fig. 7 for extreme inclusions in the wires of the five different materials. The best-fit distribution, whose parameters have been calculated with the moment's method described by Beretta et al. [25], is plotted on the same graph together with $95 \%$ confidence bands. It can be clearly seen that the Gumbel distribution describes quite well the data. The parameters of the distribution of extreme inclusions for the five different families are reported in Table 4.

In particular, the maximum defect with a return period $\mathrm{T}$ (the maximum defect which is expected to be exceeded once every $\mathrm{T}$ observations) can be calculated as follows:

$d_{\max }(T)=\hat{\lambda}+\hat{\delta}(-\log (-\log (1-1 / T)))$

The different datasets were analyzed according to this procedure, and the prospective size of maximum defects for $T=1000$ and $T=10,000$ (obtaining the defects that are exceeded once every 1000 and 10,000 specimens) was calculated. In this way, we are estimating extreme defects that should be correlated to the lower bound of fatigue limit.

\section{Empirical Correlation Between Fatigue Limit and Extreme Inclusions}

The fatigue strength of a component containing defects or inhomogeneities subjected to axial or bending forces can be predicted on the basis of the models of Murakami and Endo [26]. They showed that at the fatigue limit, small, nonpropagating cracks are always present at a defect tip. Because of this, the inhomogeneities can be treated as cracks and accordingly the fatigue limit $\left(\Delta \sigma_{\text {lim }}\right)$ has to be calculated as the cyclic threshold stress at which the cracks emanating from the defects do not propagate.

This type of analysis suggests the need to calculate the stress intensity factor (SIF or K) for a crack with an irregular shape. Murakami suggested that the maximum stress intensity for a crack with an irregular shape could be calculated easily with the simple equation:

$K=0.65 \sigma \sqrt{\pi \sqrt{\text { area }}}$

where $\sqrt{ }$ area is the square root of the defect area projected on a plane perpendicular to the applied stress. Because of this model, in the following description, the inclusions size $d$ will be expressed in terms of $\sqrt{ }$ area. The prediction of fatigue limit would therefore be possible considering at the fatigue limit $\Delta K=\Delta K_{\text {th }}$ and then

$\Delta \sigma_{\lim }=\frac{\Delta K_{\mathrm{th}}}{0.65 \sqrt{\pi d}}$ 
Fig. 7 a Gumbel probability plot for the inclusions at fracture origin. b Magnification of the overlapping region (Color figure online)
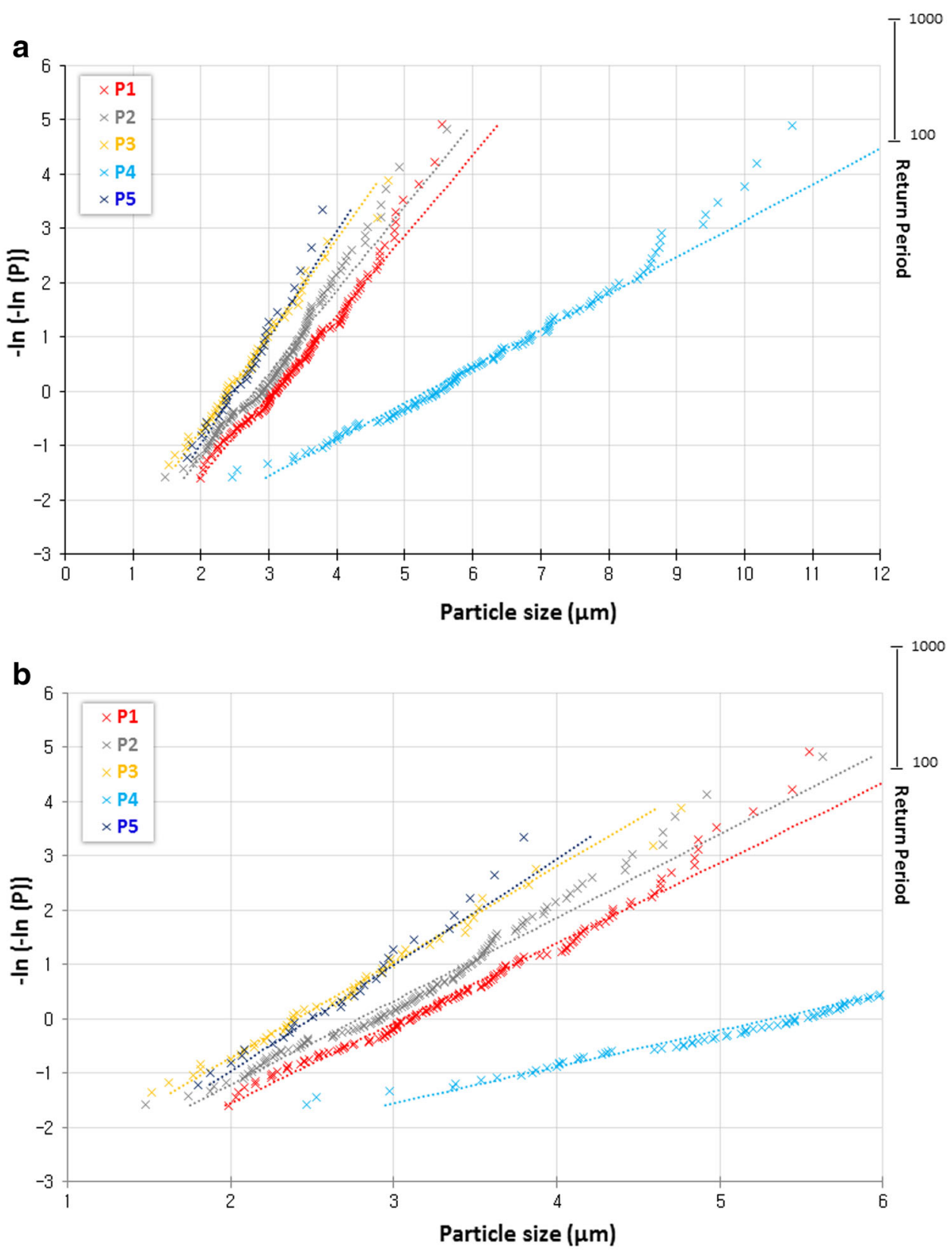

Table 4 Fitted parameters of the Gumbel distribution for the five materials and expected particle size for a return period $T=10^{3}$

\begin{tabular}{lllc}
\hline Material & $\lambda(\mu \mathrm{m})$ & $\delta(\mu \mathrm{m})$ & $d_{\max }(\mu \mathrm{m})$ \\
\hline P1 & 2.73 & 0.68 & 7.27 \\
P2 & 2.61 & 0.58 & 6.60 \\
P3 & 1.83 & 0.48 & 5.14 \\
P4 & 4.57 & 1.43 & 14.46 \\
P5 & 2.51 & 0.54 & 6.92 \\
\hline
\end{tabular}

However, the application of Eq. (8) is not straightforward since the fatigue threshold is a function of crack or defect size [26] and only in the case of along crack does it trend to a constant value $-\Delta K_{\mathrm{th}}$, IC. A conservative approach, proposed by Robertson et al. [27], is to estimate the $\Delta K_{\text {th }}$ for small cracks in terms of a lower bound of the $\mathrm{d} a /$ $\mathrm{d} N$ curves obtained on standard CT specimens. According to this approach, the fatigue limit for small cracks is

$\Delta \sigma_{\lim }=\frac{\Delta K_{\mathrm{th}, \mathrm{IC}, \text { lowerbound }}}{0.65 \sqrt{\pi d}}$

A better alternative is to model the $\Delta K_{\mathrm{th}}-d$ (where $d$ is the defect/crack dimension) relationship, otherwise called Kitagawa-Takahashi diagram, by experimental tests [26] or theoretical models (see [21] for a review). In both approaches, the result will be a relationship of the type: 
$\Delta \sigma_{\lim }=\frac{C_{1}}{\sqrt{\text { area }}^{1 / n}}$,

where $n>2$ (this condition is necessary for describing the tendency $\Delta \sigma_{\text {lim }} \rightarrow \Delta \sigma_{\text {wo }}$, see Fig. 6). According to $\mathrm{Mu}-$ rakami and Endo [21], the parameter $C_{1}$ depends on the metal matrix hardness HV (or, in other words, to the tensile properties of the material) and $n=6$ for $d<1000 \mu \mathrm{m}$, while for Robertson $n=2$ and $C_{1}=\Delta K_{\mathrm{th}}$, lower bound.

Considering that the strain amplitudes corresponding to fatigue lives greater than $10^{5}$ are in the region of nominally elastic response for NiTi SMA $\left(\Delta \varepsilon_{\text {lim }} \alpha \Delta \sigma_{\text {lim }}\right)$, it is assumed that in the region of fatigue limit, a relationship of the type:

$\Delta \varepsilon_{\lim }=\frac{C_{2}}{\sqrt{\operatorname{area}}^{1 / n}}$

should be valid. In the following, the existence of such a relationship will be shown. The parameter $C_{2}$ should be a function of the tensile properties; the different wire classes have very similar static properties, so it appears correct to apply Eq. (11) to the experimental evidence.

The inclusion dimensional distribution can be related to the fatigue performances of different materials by plotting the expected maximum diameter $(T=1000)$ against the fatigue limit (as in Fig. 8). According to this figure, samples obtained from materials characterized by different inclusion's distributions behave differently in fatigue testing, extreme inclusion dimensions and fatigue limit are anti-correlated (thus confirming Eq. 11). This result supports the concepts of extreme value inclusion rating for the quality control of NiTi-based shape memory materials.

Material P5 appears as outlier, or it seems not to follow perfectly the expected trend. This behavior can suggest that maximum inclusions size might reasonably not be the only factor affecting fatigue. Since P5 has the smallest area fraction of inclusions, the data imply that the total number of inclusions (and consequently the probability of having a critical inclusion at the highest strain location) could be an additional contributing factor, albeit small compared to the maximum size which seems to dominate.

\section{Model for the Effect of Inclusions on Fatigue of NiTi Wires}

In this section we propose a fatigue prediction model exploiting the theory presented in "Empirical Correlation Between Fatigue Limit and Extreme Inclusions" Section. The model assumes the fatigue failure occurs when a crack, propagating from a starting defect, such as an inclusion, reaches a critical value that we arbitrarily set to half wire diameter.

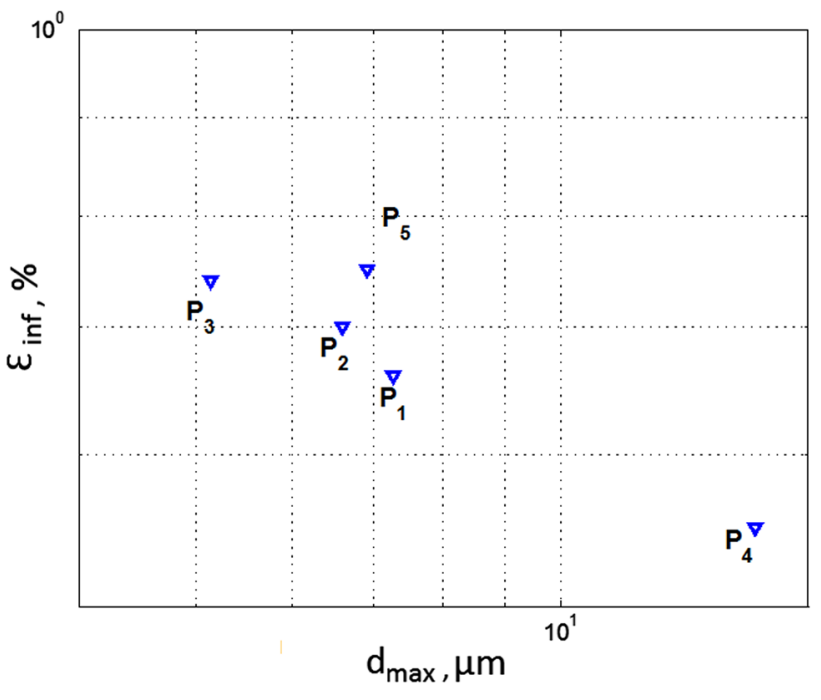

Fig. 8 Empirical correlation between $d_{\max }$ (estimated with extreme value distribution) and $\varepsilon_{\text {inf }}$ for the Nitinol wires

In order to evaluate the crack propagation, we chose the NASGRO propagation equation taking into account the ElHaddad correction for small crack.

\section{Fatigue Strength Model}

Another alternative is the El-Haddad model [28] which describes the peculiar shape of the Kitagawa-Takahashi diagram assuming that an inherent fictitious defect size $d_{0}$ should be added to the real defect/crack. This inherent fictitious defect size (also called the El-Haddad parameter) is the defect size that would exist at the cyclic stress $\Delta \sigma_{\text {wo }}$ (fatigue limit of the material) if the threshold was $\Delta K_{\mathrm{th}, \mathrm{IC}}$ :

$d_{0}=\frac{1}{\pi}\left(\frac{\Delta K_{\mathrm{th}, \mathrm{IC}}}{0.65 \Delta \sigma_{\mathrm{wo}}}\right)$

This formula, firstly adopted by Beretta et al. [29], is different from the original El-Haddad's model [28], since it refers to Eq. (7) for calculating the stress intensity factor (SIF), so that the El-Haddad parameter is expressed in

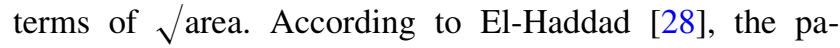
rameter $d_{0}$ should then be added to the defect size in order to calculate the fatigue limit in the presence of a given defect $d$ with Eq. (8) obtaining:

$\Delta \sigma_{\lim }=\Delta \sigma_{\mathrm{wo}} \sqrt{\frac{d_{0}}{d+d_{0}}}$

Accordingly, the threshold depends on defect size as

$\Delta K_{\mathrm{th}}=\Delta K_{\mathrm{th}, \mathrm{IC}} \sqrt{\frac{d}{d+d_{0}}}$

The advantage of the El-Haddad model is that it allows one to easily describe the smooth transition of $\Delta K_{\mathrm{th}}$ toward $\Delta K_{\text {th, IC }}$ (or alternatively $\Delta \sigma_{\text {lim }} \rightarrow \Delta \sigma_{\text {wo }}$ for $d \rightarrow 0$ ). 
On the other hand, this model requires some tests with small defects for determining the parameters of Eq. (14). The functional relationship $\Delta \sigma_{\mathrm{lim}}-d$ remains Eq. (10), but here $n$ is variable within $2 \leq n<\infty$ for $0 \leq d<\infty$.

\section{Threshold and Crack Growth Data for NiTi}

Robertson \& Ritchie [30] obtained crack growth data for NiTi with tests carried out at $37{ }^{\circ} \mathrm{C}$ at different load ratios ( $R=0.1, R=0.5$, and $R=0.7$ ) on CT specimens, obtaining threshold values reported in Fig. 9a. Considering that the effect of the crack-closure for linear elastic fracture mechanics (LEFM) tests can be described by Schijve's equation [31]:

$\frac{\Delta K_{\mathrm{eff}}}{\Delta K}=U=0.55+0.33 R+0.12 R^{2}$,

where $R$ is the stress ratio, it is the possible to estimate $\Delta K_{\text {th, eff }}$ and then $\Delta K_{\text {th }}$ can be calculated at the different stress ratios as

$\Delta K_{\mathrm{th}}=\frac{\Delta K_{\mathrm{th}, \mathrm{ef} f}}{U}$

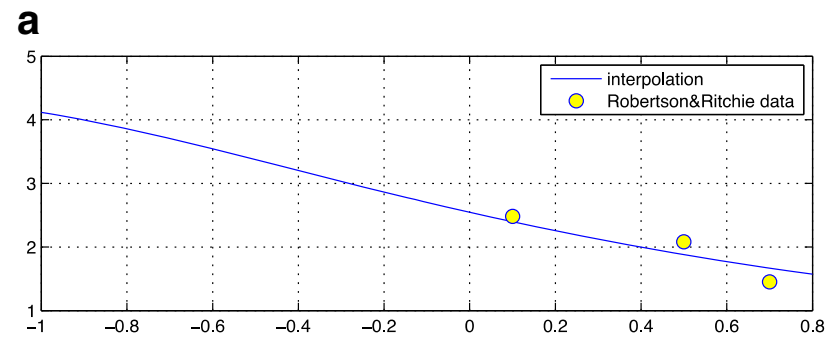

b

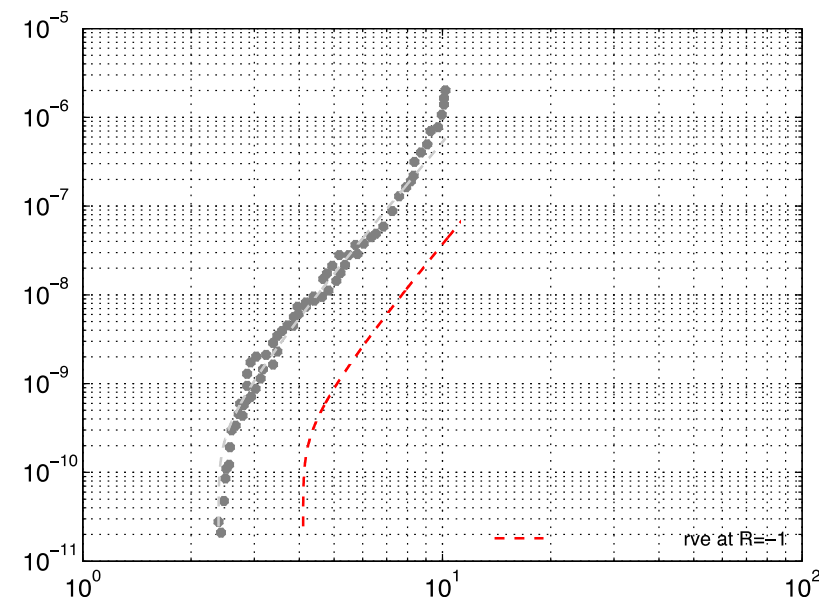

Fig. 9 Estimation of crack growth data at $R=-1$ : a threshold data by Robertson and Ritchie [12] and estimation of dependence of $\Delta \mathrm{Kth}$ with $R ; \mathbf{b}$ data by Robertson at $R=0.1$ and estimated growth curve at $R=-1$ with NASGRO equation
As seen in Fig. 9a, the approximation is acceptable and the estimated $\Delta K_{\text {th }}$ at $R=-1$ is $4.0 \mathrm{MPa} \sqrt{ }$. Corresponding the concepts of Robertson \& Ritchie [30], the lower bound for fatigue propagation of small cracks can be estimated to be $\Delta K_{\text {th, lower bound, } R=-1}=2.2 \mathrm{MPa} \sqrt{ } \mathrm{m}$.

A similar analysis has been carried out for estimating the crack growth curve at $R=-1$. In particular, the Robertson's data at $R=0.1$ have been interpolated with the expression for (equivalent to the NASGRO propagation equation [32]):

$\frac{\mathrm{d} a}{\mathrm{~d} N}=C \Delta K^{n}\left(1-\frac{\Delta K_{\mathrm{th}, \mathrm{IC}}}{\Delta K}\right)^{p}$

Then, the equation parameters were adjusted according to Eq. (15), obtaining the estimated growth curve shown in Fig. 9b.

\section{Fatigue Strength Model Versus Experimental Tests}

Considering the data concerning the defects of the P4 material tested at $37{ }^{\circ} \mathrm{C}$, the maximum strain level at which there are run-outs is $0.7 \%$ and it is related to the presence of the smallest inclusions. Assuming that the Young's modulus for all the wire batches is $E=68,000 \mathrm{MPa}$, it can be estimated that $\Delta \sigma_{\mathrm{wo}}=952 \mathrm{MPa}$. This estimate was confirmed by a series of cyclic strain-control experiments at $R_{\varepsilon}=-1$, that clearly showed that the limit for a cyclic elastic response corresponds to $\Delta \varepsilon=1.4 \%$. So, it is then possible to simply calculate $d_{0}$ with Eq. (12) and then the relationship between fatigue limit and inclusion size (Eq. 13).

A series of extra experiments were then carried out on P4 wires for confirming this prediction by applying the following procedure:

i) Wires were tested at the strain level of $0.55 \%$ and the inclusions at fracture origin of broken wires were measured under SEM;

ii) The run-out wires were then re-tested at higher strain amplitudes $(0.60$ and $0.65 \%)$ in order to break them and their fracture services were examined under SEM in order to detect the inclusion at fracture origin.

In this way, it was possible to clearly determine the relationship between defect size and fatigue limit (expressed in terms of $\Delta \sigma_{\mathrm{w}}$ ) and compare these precise data with the prediction by the El-Haddad model, as shown in Fig. 10a. It can be clearly seen how the model nicely predicts the fatigue strength for $\mathrm{P} 4$ wires, which have the lowest fatigue strength.

The model is compared with all the wire fatigue failed at strain levels close to the fatigue limit (failures with $\varepsilon \leq \varepsilon_{\mathrm{D}}$ ) 

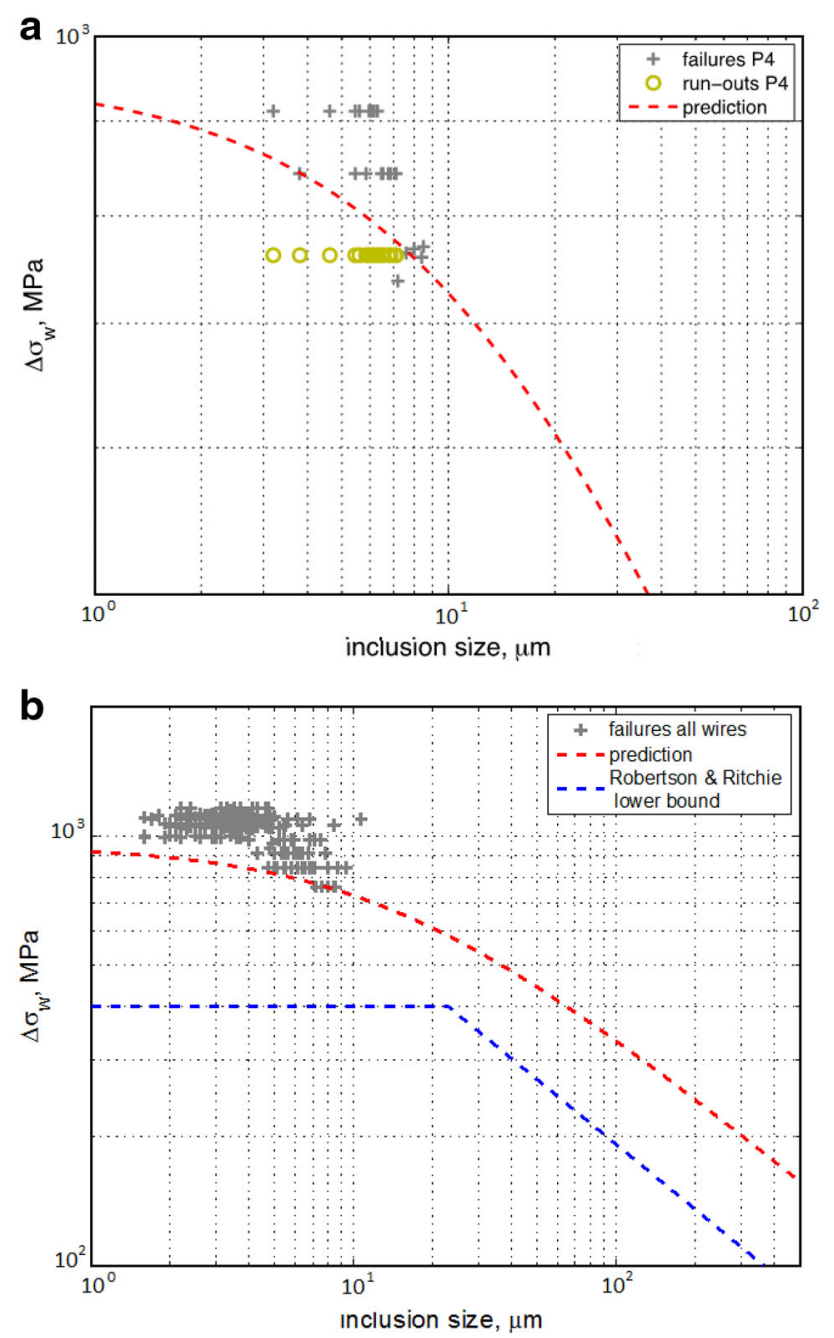

Fig. 10 The estimated Kitagawa diagram at $R=-1$ for NiTi wires compared with experimental data: a comparison with $\mathrm{P} 4$ data at the fatigue limit (run-out wires have been re-tested at higher strain amplitudes); b comparison with broken wires of the other batches (Color figure online)

in Fig. 10b, where it is possible to also see that the model conservatively predicts the lower bound of the failures for the other wire batches. In the same graph, it can be seen that the lower bound limit for fatigue failure by Robertson $\&$ Ritchie is very conservative with respect to actual failures in the fatigue limit region.

\section{Life Prediction}

The relationship $\mathrm{d} a / \mathrm{d} N-\Delta K$ can be also integrated in order to estimate fatigue life. As for the SIF, it is reasonable to assume that the cracks propagate with a nearly semi-circular shape, and therefore the SIF solution at the crack tip, for crack propagation in the wires subjected to rotating bending, was expressed as follows:

$\Delta K=F \cdot \Delta S \cdot \sqrt{\pi a}$,

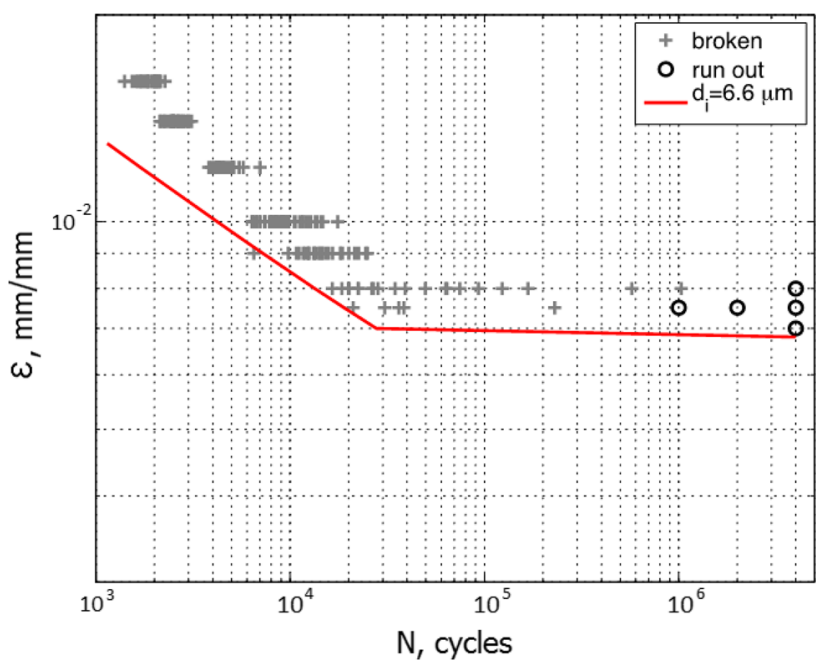

Fig. 11 Conservative life prediction for P2 wires considering the initial defect with return period $T=1000$

where $a$ is the crack depth and $F$ has been taken from Carpinteri et al. [33] (the difference between Eqs. (7) and (17) for a semi-circular cracks is a few percents when the crack depth is less than $5 \%$ of wire diameter).

The fatigue life of the wires was estimated with a simple crack propagation model, with these inputs:

i) The propagation crack growth rate (Eq. 16) estimated at $R=-1$ has been modified by expressing $\mathrm{d}_{0}$ in terms of crack depth;

ii) For the initial defects: initial defect $d_{i}$ was calculated from the LEVD distribution as the defect size with $T=1000$;

iii) The final crack size at failure was assumed to be $50 \%$ of the wire diameter;

The predicted S-N diagram for the $\mathrm{P} 2$ wires is shown in Fig. 11. It can be seen that predictions are very close to experimental data. Similar good predictions were also obtained for the other wires.

\section{Conclusions}

In this work, results and analysis of RBT fatigue testing on $300-\mu \mathrm{m}$ diameter superelastic NiTi wires obtained from five different processes are presented and discussed. Results of the research can be summarized as follows:

- Cycles to failure of about 1500 samples from five materials were recorded for several deformation levels and a descriptive model of $\varepsilon-N$ behavior was utilized to compare the materials. A consistent bilinear description of the fatigue diagrams both in the finite life and fatigue limit region was given. 
- Fracture surfaces of failed wires were inspected by FESEM and inclusions at the crack nucleation site measured, classified, and analyzed with the concepts of 'statistics of extremes.' These extreme inclusions were successfully correlated to the lower bound of strains at fatigue limit. Wires characterized by LEVD distributions predicting smaller inclusions provide better fatigue performance.

- Apparently, not the same correlation between maximum inclusions size and fatigue life can be found by analyzing the longitudinal metallographic samples as prescribed by ASTM F2063. The empirical correlation between lower bound of fatigue limit and extreme inclusions was confirmed by a fatigue strength prediction based on the El-Haddad model, which is a conservative estimate of fatigue strength for the different wire batches.

- Finally, a fatigue life prediction model was proposed. The relationship between $\mathrm{d} a / \mathrm{d} N-\Delta K$ has been integrated for estimating the fatigue life. Results show a good agreement between the experimental data and the predicted fatigue lives.

Additional research is in progress to extend the statistical data presented in this work and further investigate fatigue behavior in Nitinol. Moreover, since the extreme inclusions were shown to be strongly correlated with fatigue performance, actions have been undertaken for the application of extreme value inclusion rating to NiTi SMA wires.

\section{References}

1. Melton N, Mercier O (1979) Fatigue of NiTi thermoelastic martensites. Acta Metall 27:137-144

2. Adler PH, Allen J, Lessar J, Francis R (2007) Martensite transformations and fatigue behavior of nitinol. J ASTM Int 4:3-16

3. Pelton AR (2011) Nitinol fatigue: a review of microstructures and mechanisms. JMEPEG 20:613-617

4. Reinoehl M, Bradley D, Bouthot R, Proft J (2000) The influence of melt practice on final fatigue properties of superelastic NiTi wires. In SMST-2000: Proceedings of the International Conference on Shape Memory and Superelastic Technologies, pp 397-403

5. Sawaguchi T, Kaustrater G, Yawny A, Wagner M, Eggler G (2003) Crack initiation and propagation in 50.9 at. pct Ni-Ti pseudoelastic shape memory wires in bending-rotation fatigue. Metall Mater Trans A 34A:2847-2860

6. Wong S, Lin ZC, Tahran A, Boylan J, Pike K, Kramer-Brown P (2009) An investigation of factors impacting nitinol wire fatigue life. J ASTM Int 6:99-107

7. Schaffer JE, Plumley DL (2009) Fatigue performance of nitinol round wire with varying cold work reductions. J Mater Eng Perform 18:563-569

8. Rahim $\mathrm{M}$ et al (2013) Impurity levels and fatigue lives of pseudoelastic NiTi shape memory alloys. Acta Mater 61(10):3667-3686

9. Steegmuller R et al (2014) Analysis of new nitinol ingot qualities. J Mater Eng Perform 23:2450-2456
10. Launey M et al (2014) Influence of microstructural purity on the bending fatigue behavior of VAR-melted superelastic nitinol. J Mech Behav Biomed Mater 34:181-186

11. Standard specification for wrought nickel-titanium shape memory alloys for medical devices and surgical implants, ASTM F206312,2012

12. Standard practice for extreme value analysis of nonmetallic inclusions in steel and other microstructural features, ASTM E2283-08, 2014

13. Murakami Y (2002) Metal fatigue: effect of small defects and nonmetallic inclusions. Elsevier, Oxford

14. Standard test method for transformation temperature of nickeltitanium alloys by thermal analysis, ASTM F2004-05, 2010

15. Standard test method for determination of transformation temperature of nickel-titanium shape memory alloys by bend and free recovery, ASTM 2082-03, 2003

16. Standard test method for tension testing of nickel-titanium superelastic materials, ASTM F2516-14, 2014

17. Sczerzenie F, Vergani G, Belden C (2012) The measurement of total inclusion content in nickel-titanium alloys. J Mater Eng Perform 21:2578-2586

18. Spindel JE, Haibach E (1979) The method of maximum likelihood applied to the statistical analysis of fatigue data. Int $\mathbf{J ~ F a - ~}$ tigue 1:81-88

19. Standard practice for statistical Analysis of linear or linearized stress-life (S-N) and strain-life $(\varepsilon-N)$ fatigue data, ASTM E73910,2010

20. Nelson W (1982) Applied life data analysis. Wiley, New York

21. Murakami Y, Endo M (1994) Effect of defects, inclusions, and inhomogeneities on fatigue strength. Int J Fatigue 16:163-182

22. Murakami Y, Toryiama T, Coudert EM (1994) Instructions for a new method of inclusion rating and correlation with the fatigue limit. J Test Eval 22:318-326

23. Murakami Y, Beretta S (1999) Small defects and inhomogeneities in fatigue strength: experiments, models and statistical implications. Extremes 2:123-147

24. Coles S (2001) An introduction to statistical modeling of extreme values. Springer, London

25. Beretta S, Murakami Y (1998) Statistical analysis of defect for fatigue strength prediction and quality control of materials. Fatigue Fract Eng Mater Struct 21:1049-1065

26. Murakami Y, Endo M (1986) Effect of hardness and crack geometries on $\Delta \mathrm{K}$ th of small cracks emanating from small defects, In: The Behavior of Short Fatigue Cracks, EGF Pub. 1, pp 275-293

27. Robertson SW, Ritchie RO (2007) A fracture-mechanics-based approach to fracture control in biomedical devices manufactured from superelastic nitinol tube. J Biomed Mater Res-Part B 84: 26-33

28. El-Haddad MH, Smith KN, Topper TH (1979) Fatigue crack propagation of short cracks. J Eng Mater Tech 101:42-46

29. Beretta S, Ghidini A, Lombardo F (2005) Fracture mechanics and scale effects in the fatigue of railway axles. Eng Fract Mech 72:195-208

30. Robertson SW, Stanliewicz J, Gong XY, Ritchie RO (2006) In Mertmann M (ed) SMST 2003: Proceedings of the International Conference on shape memory and superelastic technologies, Baden-Baden, Germany, ASM Intl., Materials Park, OH, 2006, pp 79-88

31. Schijve J (2001) Fatigue of structures and materials. Kluwer Academic Publishers, Dordrecht

32. Fatigue crack growth computer program NASGRO version 3.0Reference Manual. Rapp. Tech. JSC-22267, NASA, 2001

33. Carpinteri A (1994) Propagation of surface cracks under cyclic loading. Handbook of fatigue crack propagation in metallic structures. Elsevier, Amsterdam, pp 653-705 